\title{
FACTORES SOCIOECONÓMICOS Y SU REPERCUSIÓN EN EL PROCESO EDUCATIVO DE ESTUDIANTES UNIVERSITARIOS
}

\author{
SOCIOECONOMIC FACTORS AND THEIR IMPACT ON THE \\ EDUCATIONAL PROCESS OF COLLEGE STUDENTS
}

\author{
Mónica Tumbaco \\ mtumbaco@upse.edu.ec \\ Francisco Villón \\ fvillon@upse.edu.ec \\ Universidad Estatal Península de Santa Elena - UPSE, Ecuador.
}

\section{RESUMEN}

El abandono universitario por factores socioeconómicos es un fenómeno muy común en la mayoría de los países latinoamericanos, razón por la cual se viene planteando como uno de los problemas prioritarios a ser investigado y adecuadamente tratado. En ese sentido, el Instituto Internacional de la UNESCO para la Educación Superior en América Latina y el Caribe (IESALC) plantea la necesidad de realizar estudios en toda la región, que ofrezcan luz sobre los factores causales y la búsqueda de solución al problema. La Educación Superior, debe formar personas calificadas en el manejo y conocimiento de los procesos tecnológicos de los diferentes sectores productivos, supone además, acrecentar capacidades como pensar creativa y críticamente, tomar decisiones en la solución de problemas, capacidad de aprendizaje, capacidad de pensamiento práctico, capacidades de organización, creatividad, responsabilidad, liderazgo y valores para participar de manera eficiente en el desarrollo de la economía nacional.

Palabras clave: economía, sociedad, proceso educativo, estudiantes, universidad.

\begin{abstract}
The university drop its dimensions, it is a very common phenomenon in most Latin American countries, why, has been established as one of the priority issues to be investigated and properly treated. In this regard, the UNESCO International Institute for Higher Education in Latin America and the Caribbean (IESALC) proposesthe need for studies throughout the region, offering light on the causal factors and the search for solution to the problem. Higher education must be qualified in the management and knowledge of the technological processes of the different productive sectors people also supposed to increase capacities as thinking creatively and critically, make decisions in solving problems, learning ability, ability to practical thinking, organizational skills, creativity, responsibility, leadership and values to participate efficiently in the development of the national economy.
\end{abstract}

Keywords: economy, society, educational process, students, university.

Recibido: 4 de octubre de 2016

Aceptado: 23 de mayo de 2017

Publicado: 30 de junio de 2017

\section{INTRODUCCIÓN}

La educación es una necesidad vital para el desarrollo nacional y modernización del sistema educativo, o el mejoramiento de la calidad de los servicios educativos, por lo que es necesario que, entre otros aspectos se prioricen los siguientes:

- La enseñanza debe estar basada a la realidad concreta y del futuro previsible, de modo que el alumno pueda ubicarse en el mundo real, encarar sus desafíos y lograr ser productivo.

- Renovar los programas curriculares según las exigencias técnicos pedagógicos modernos.

- Incursionar en el mercado ofertando bienes y servicios que además de generar entes, sirvan de prácticas profesionales.

Teobaldo (1996) en su investigación cuantitativa y cualitativa en estudiantes del ciclo básico de la Universidad de Buenos Aires, señala que el disloque de los estudiantes en su condición de 
alumno, se debe a que no aprende el "oficio de estudiante", el cual consiste en que el aprendizaje requiera adaptarse a nuevos estilos y modelos de docentes, diferentes normativas y funcionamiento institucional. Asimismo, los propios estudiantes confiesan que tienen dificultades en la capacidad de síntesis, en la forma de estudiar, en la comprensión de textos y en el uso de la lengua oral y escrita. Por ello, el fracaso (abandono de una institución), es una construcción que el estudiante realiza y que está determinado por el medio socioeconómico y psíquico en el que se desenvuelve.

Asimismo, posibilitar en los egresados y los trabajadores, el desarrollo de capacidades que les permitan su inserción y reinserción en el mercado laboral, de manera dependiente o utilizando elementos de gestión empresarial, con actitud y capacidad emprendedora y apostando por el autoempleo en un mercado competitivo. Este nuevo contexto, exige cambios en la forma de gestión de los centros de formación, en base a un trabajo conjunto entre los agentes educativos y el entorno productivo, constituyendo así un equipo comprometido con los lineamientos y metas educacionales y los requerimientos de este entorno. En función a lo descrito anteriormente, los rasgos que caracterizan a la educación superior son:

\section{a. Desempeño profesional competente.}

Formar personas que respondan a las necesidades de un trabajo productivo de calidad, desarrollando capacidades para el manejo tecnológico con eficiencia y responsabilidad, incorporando aspectos actitudinales e instrumentales que permitan el trabajo en equipo, capacidad de aprendizaje, tomar decisiones con rapidez en situaciones de presión o contingencia y ser polivalentes.

b. Mayor movilidad y adaptabilidad laboral. Formar profesionales técnicos altamente calificados, capaces de responder a los distintos requerimientos a lo largo de su vida profesional; que impulsen la reconversión productiva de las empresas, su inserción en el mercado laboral nacional, e internacional y el crecimiento sostenible del país en una perspectiva del desarrollo humano; personas capaces de actualizarse permanentemente de manera autónoma, adecuarse y responder asertivamente a los cambios tecnológicos y del mercado laboral.

c. Flexibilidad de la formación. Brindar a los distintos beneficiarios de la Educación superior la posibilidad de adquirir capacidades terminales organizadas en módulos, permitiendo, por un lado, tener opciones de empleabilidad durante su formación, y por otro, la posibilidad de reinsertarse en el sistema formativo, si éste fuera el caso.

La igualdad de todos para tener acceso a la educación, no es real. No se puede ocultar las desigualdades sociales existentes. Como tal, quien tiene recursos económicos, a su vez tiene derecho a mejor educación para sus hijos. Los trabajadores del sector medio, solo pueden alcanzar los niveles inferiores del sistema educativo. Esta discriminación se hace evidente de diversas maneras: un grueso sector de la población en edad escolar es cada vez creciente y provienen de los sectores más pobres los que abandonan el estudio por falta de recursos económicos, trabajo, problemas sociales A partir de ello surgen problemas sociales de bajo rendimiento académico, deserción, repitencia, que es el caso concreto de los sectores más deprimidos y periféricos de las ciudades y forman parte de la exclusión social.

En las dos últimas décadas se viene agudizando esta situación por la continua crisis económica que vive el país, que ha originado entre otras situaciones el notorio crecimiento del desempleo y subempleo; y por ende, la educación superior es limitada en los adolescentes y jóvenes estudiantes, por las situaciones críticas en lo económico, trabajos no estables y estadías elevadas en su precio.

En consecuencia, la población en general sufre un gran cambio en el aspecto económico como en el laboral. El alto desempleo ha intensificado la competencia por trabajo de todo tipo, haciendo que las personas mayores y los jóvenes sean cada vez más marginadas de todas las oportunidades de trabajo y ocupación de cargos importantes.

El déficit de desarrollo intelectual de los estudiantes, no sólo por la inestabilidad familiar en los hogares, ya sea porque su asistencia a clases es irregular, porque tienen que trabajar, porque están desmotivados, porque los maestros son deficientes, porque los métodos de enseñanza-aprendizaje son obsoletos, porque no hay libros o por cualquier causa que impida el ingreso y la permanencia en los niveles de la educación sistemática, dejan en la preparación académica de cada estudiante una serie de intervalos de discontinuidad que persisten a medida que avanza en los grados del sistema educativo. Por humanismo, los estudiantes del sector público deberían recibir la mejor educación o al menos similar a la privada, para abrirles más oportunidades de trabajo productivo, con mayores perspectivas de progreso, ya que este contingente también constituye un amplio sector y 
un valioso material potencial de desarrollo económico y social del país.

\section{Variables del proceso educativo}

El escenario mundial está signado por la globalización, fenómeno económico y social caracterizado por la velocidad en las comunicaciones, acelerado desarrollo científico y tecnológico, y un sistema de mercado tanto de productos como servicios, alentado por las transnacionales. En este contexto, se desarrolla la sociedad del conocimiento, en el cual el ser humano se convierte en el centro de atención, a quien se le demanda un perfil basado en el dominio de capacidades como pensamiento crítico y creativo, que ayudan a tomar decisiones con rapidez aún en situaciones de contingencia; solucionar problemas; trabajar en equipo, capacidad de aprender con rapidez, ser multilingüe y multihábil.

Los ajustes estructurales que conlleva al deterioro de la estabilidad social y económica manifestándose en el aumento desmedido de la pobreza en las zonas rurales; a pesar de que el actual gobierno considera a éste como tema de primer orden y propone y lleva a cabo una reforma económica, lo que debe ir de la mano con la reforma social.

Los indicadores que tienen influencia en el retraso suelen ser el ausentismo, deserción, bajo rendimiento académico, la posición económica del estudiante, estado civil, orientación vocacional, ingresos que perciben, procedencia rural - urbano, e incluso el tipo de vivienda para los fines del éxito y vida adecuada.

También se debe considerar que un importante número de estudiantes, siempre señalarán como causas de su abandono universitario situaciones externas o ajenas a su responsabilidad, ya que la responsabilidad propia y la palabra 'fracaso', son difíciles de asimilar.

Específicamente para Otero, algunas de las variables que influyen en el proceso educativo son los factores psicosociales $y$ sociodemográficos del contexto familiar, recursos económicos, alguna limitación física y mental, la ausencia de disciplina y el método en el estudio, aunado a esto se encuentran deficiencias en los programas académicos, las expectativas sobre la carrera matriculada, la matrícula en carreras no deseadas, la carga académica, el lugar de residencia, oportunidades académicas, la falta de orientación vocacional, complejos componentes de la personalidad (aspiraciones, motivación, entre otros).

\section{Factores sociales}

Brea, M. (2005), resalta en su investigación los factores que por su importancia influyen directamente en el proceso educativo, entre estos se encuentran los aspectos sociales, como son las condiciones laborales, desorientación vocacional, baja autoestima y la posible precaria adaptación al medio universitario.

En el aspecto social se encuentran como factores influyentes, los problemas familiares, los embarazos en las jóvenes, el estado civil y la falta de orientación vocacional.

Problemas Familiares. Este factor está relacionado con la problemática en general que atraviesan los hogares de los estudiantes, es una situación que se vuelve compleja cuando no se encuentran soluciones inmediatas. Se provoca por la separación familiar, la violencia intrafamiliar, los problemas de salud, el cuidado de sus hijos en los primeros meses de vida, la migración, entre otras razones.

Casarse o unión de hecho. Es una institución social que crea un vínculo conyugal entre sus miembros. Este lazo es reconocido socialmente, ya sea por medio de disposiciones jurídicas o por la vía de los usos y costumbres. El matrimonio establece entre los cónyuges, y en muchos casos también entre las familias de origen de éstos, una serie de obligaciones y derechos que también son fijados por el derecho, que varían, dependiendo de cada sociedad. De igual manera, la unión matrimonial permite legitimar la filiación de los hijos procreados o adoptados de sus miembros, según las reglas del sistema de parentesco vigente.

Embarazos o maternidad. Los embarazos en las jóvenes es otra de las causas que aumentan los problemas sociales, estos se dan por la independencia que adquieren muchas de ellas de su núcleo familiar, otras deciden tener hijos, y en última instancia, la falta de información familiar e institucional sobre la educación sexual para evitar embarazos a temprana edad.

Orientación vocacional. La falta de orientación vocacional o falta de mayor información de la carrera a estudiar es una causa predisponente del estudiante, asociándola con las situaciones sociales que vivió antes de ingresar a la universidad.

Orientación profesional. Es la elección de una profesión y/o trabajo, apunta no sólo hacia una 
actividad u opción profesional, sino a una forma de vida, por lo tanto, la elección debe hacerse consciente de que con ella formamos parte de nuestra identidad, de nuestro "yo" y que a través de ella, asumimos un rol (Aguirre Baztán, 1996).

\section{Factores económicos}

Ruiz (2007), plantea que el estudiantado es afectado en su proceso educativo, por no contar con suficientes recursos económicos para sostener la familia y sus estudios, además de proceder de hogares en condiciones socioeconómicos desfavorables. Resalta en su estudio el factor económico, el que está estrechamente relacionado con el familiar y el laboral. En algunos casos el estudiante siente que no tiene tiempo para dedicarse al estudio y trabajo, aparte de otros compromisos.

De los Santos (2005), refiere en su estudio que, el origen del abandono fue la necesidad de trabajar para garantizar su estabilidad económica, lo que les restó capacidad de dedicación exclusiva para estudiar (apenas estudiaban unas horas cuando podían).

Dependencia económica familiar. Otra de las causas fue la falta de apoyo familiar, se expresa en la imposibilidad de brindarles apoyo económico a sus hijos, debido al problema de empleo y los niveles de pobrezas que muchas familias tienen.

Ocupación de los estudiantes. Las ocupaciones laborales tienen su influencia positiva, porque satisface algunas necesidades económicas desde el punto de vista personal y familiar, y negativa porque no tomaron en cuenta el horario de salida del trabajo con el de la entrada a clases en la universidad.

Desempleo. El desempleo es uno de los factores relevantes, éste afecta una gran parte de la población, las familias no cuentan con ingresos estables que les permita resolver su problemática económica, lo que influye en los jóvenes que optan por estudiar en la universidad, muchos se limitan a no ingresar y quienes lo hacen se mantienen por un corto periodo por la falta de recursos económicos.

\section{Proceso educativo}

Tradicionalmente se ha planteado el proceso educativo como la relación que se establece entre el enseñar y el aprender, como si se tratase de una relación de causa-efecto, el profesor enseña (trasmite) contenidos que deben ser aprendidos (memorizados) por el estudiante. Esta visión mecánica y reduccionista del proceso educativo ha sido desmentida, en la actualidad, por factores del nuevo contexto y, particularmente por los resultados, en general poco satisfactorios, que los estudiantes logran en los diversos programas de formación.

En las últimas décadas han surgido diversas propuestas que bajo denominaciones como aprender a aprender 0 aprender a pensar, expresan nuevas intenciones del sistema y de las instituciones educativas con relación a lo que debe esperarse de los estudiantes al concluir su proceso educativo. Evidentemente, esos propósitos plantean la necesidad de enseñar a pensar y enseñar a aprender, lo cual implica una transformación profunda de las funciones y tareas que ha desempeñado tradicionalmente el docente durante el proceso de formación. Esas expresiones proponen como objetivo educativo fundamental preparar a los estudiantes para que aprendan mejor los contenidos de los planes de estudio, pero adicionalmente, que aprendan los procedimientos para que, dentro de la institución educativa y más allá de ella, puedan continuar aprendiendo.

La expresión "aprender a aprender" no es nueva. Se introdujo al lenguaje pedagógico en la década de los setenta, cuando surgen los sistemas abiertos de enseñanza y tiene su origen en tres situaciones distintas: las teorías cognoscitivas que enfatizaban la construcción gradual del conocimiento y de sus estructuras, la conciencia de que los cambios científico-tecnológicos y sociales obligaban a un aprendizaje continuo y la convicción de que la educación debía ser conducida de manera autónoma por el propio sujeto.

Tomando como base el informe Faure (Aprender a Ser, 1972) así como otros documentos de la UNESCO en numerosos países, se incorpora la idea de que la educación es "un proceso permanente" y de que hay que propiciar el aprendizaje por cuenta propia, mediante el desarrollo de la capacidad y la actitud de seguir aprendiendo.

El concepto de aprender a aprender está relacionado estrechamente con el concepto de potenciar el aprendizaje. Aprender a aprender consiste en desarrollar las capacidades del individuo, específicamente del estudiante, a través del mejoramiento de técnicas, destrezas, estrategias y habilidades con las cuales busca acceder al conocimiento. 
El propósito de aprender a aprender debe realizarse a través de aprender a pensar, desarrollando capacidades y valores, es decir, desarrollando la cognición y la afectividad, potenciando el uso de estrategias cognitivas y metacognitivas que permitan que el sujeto logre aprendizajes significativos, los que se fundamentan en los siguientes supuestos:

- El aprendizaje se orienta hacia objetivos.

- Aprender es relacionar nueva información con conocimientos previos.

- Aprender es organizar la información.

- Aprender es adquirir un repertorio de estrategias cognitivas y metacognitivas.

- El aprendizaje, si bien se produce en etapas, no es lineal.

- El aprendizaje está influido por el desarrollo del sujeto.

- Aprender es transferir el conocimiento a nuevos problemas y contextos.

La dificultad para lograr aprendizajes significativos radica en la posibilidad de crear estructuras conceptuales organizadas y jerarquizadas para potenciar que se adquiera el conocimiento, y que lo aprendido esté disponible cuando se requiera. En otros términos, lo más relevante ya no es el saber acumulativo, sino el saber disponible para ser transferido y utilizado en diversos contextos; más importante que saber, es saber qué hacer con lo que se sabe.

El aprender a aprender y aprender a lo largo de toda la vida no significa exclusivamente la adquisición de conocimientos actualizados, sino que implica la posibilidad de tomar la iniciativa del aprendizaje, la motivación sostenida, la autoestima del sujeto, la capacidad para utilizar las diversas oportunidades de aprender que le ofrecen las instituciones formales y tradicionales de educación, incluidas las propias experiencias, la posibilidad de aprender con otros, a distancia, en escenarios distintos a los tradicionales, como resultado del desarrollo de las tecnologías de la comunicación y la información.

En la perspectiva que se aborda, pareciera otorgarse un mayor énfasis a los procesos cognitivos y a los procedimientos para aprender que a los contenidos (considerando que éstos son cada vez más abundantes y su vigencia relativa), pretenden dotar a los estudiantes de herramientas y fórmulas para mejorar sus formas de aprendizaje. Sin embargo, es importante hacer notar que tales procesos y procedimientos cognitivos, en general, no se producen en el vacío, por lo que su articulación con los contenidos disciplinarios es imprescindible.

\section{Teorías del proceso educativo}

A lo largo de la historia, la relación del maestro/a ha tenido distintas significaciones. Esa relación no la ha negado nadie. Se le ha dado más o menos importancia al maestro, pero no se ha llegado nunca a anular. Las distintas teorías responden a cuál es el elemento fundamental del proceso educativo.

- Teoría comunitario - personalista. Representantes fundamentales: Nohl y Flitmer. Lo más importante del proceso educativo es la comunidad formada por el educador (hombre maduro) y el educando (hombre en desarrollo), para que el que está en desarrollo alcance su perfección.

- Teoría de la interdependencia activa con el medio ambiente. Lo más importante es la influencia que el medio ambiente realiza sobre el hombre y lo que el hombre realiza sobre el medio ambiente. Dewey entiende por medio ambiente la suma total de condiciones que interviene y la ejecución de las actividades características del ser humano.

- Teoría de la escuela activa. El elemento fundamental es la actividad del alumno. El maestro no es anulado.

- Teoría tomista. En la doctrina de Santo Tomás, en el proceso educativo el elemento fundamental es la razón, en cuanto que ordena y dirige las operaciones humanas.

\section{Aportes teóricos de Paulo Freire}

Pedagogo brasileño del s. XX. Impregnado de la filosofía existencialista marxista, pero sobre todo representante del personalismo por el valor que otorga a la persona. Entre sus obras tenemos: "La educación como práctica de libertad", "Pedagogía del oprimido", entre otras.

La experiencia de Freire se dirige a la revolución cultural de los oprimidos y su mensaje refuerza los anclajes de la conciencia humana para la liberación.

La propuesta de Freire se dirige al desarrollo de la conciencia crítica, "concientización", ya que las transformaciones que puede ir sufriendo la conciencia, puede provocar los cambios de las sociedades. La comunicación y el diálogo sirven para la acción transformadora y la reflexión. 
Freire distingue 3 estados de conciencia que se corresponden con sociedades y culturas diferentes:

- Intransitiva: sociedades cerradas donde deciden las elites y las capas populares interpretan la realidad de manera mágica y mitológica: hombres acríticos.

- Transitiva: el hombre amplía su poder de respuesta y surgen actitudes críticas, pero a un nivel simplista y superficial.

- Crítica: con el desarrollo democrático, existe apertura al diálogo y demanda de libertad.

Este cambio se arraiga en dos tipos de educación:

- La que se llama y considera al hombre como cosa: pasividad del sujeto, mero receptor y acumulador de conocimientos.

- La que se llama liberadora y considera al hombre como persona; fundada en la actitud dialógica (el que enseña aprende del que aprende).

Este modelo fue usado por Freire en la alfabetización, con el objetivo de conocer el lenguaje y la cultura de los alfabetizandos; las formas de pensamiento popular constituyen los temas que se incorporan en el programa educativo: "concientización".

Mediante la educación, estos sujetos van descubriendo su identidad cultural, accediendo a su propia realidad.

Esta educación requiere un método de investigación donde las ciencias humanas y sociales sirven para indagar e interpretar los problemas de la realidad.

El aprendizaje del alfabeto va más allá de la lectoescritura: les lleva a distinguir las expresiones populares, la naturaleza y la cultura, a descubrir su lugar en la historia y en la sociedad, pretende afirmar la cultura despreciada.

\section{Condicionalidad de los fines educativos}

El proceso educativo se justifica en función del fin y el profesor no elige el fin, sino que colabora para que estos se realicen. Hay una serie de factores que condicionan los fines educativos.

Factores filosóficos: no es lo mismo estar en una concepción filosófica o en otra, porque cada una implica una concepción del hombre, y por lo tanto condiciona las materias y métodos educativos. Por ejemplo, los empiristas se basan en la experiencia, marxistas, personalistas, existencialistas, etc.

Factores sociales y culturales: los fines educativos están en función de la sociedad que esté implantada dicha educación, así que no es igual estar en una sociedad democrática, en una dictadura, en paz, postguerra, etc. La economía, la historia de la sociedad y la cultura condicionan los fines educativos.

Factores prácticos y utilitarios: hay que buscar un beneficio para la sociedad en la que vivimos y para el individuo en concreto. El fin educativo tiene que ser práctico y útil para estos.

Factores individuales: representado por características biológicas, procesos evolutivos y psicológicos.

\section{Propuestas de solución}

- Se considera que el aprendizaje deja de ser un proceso pasivo para ser autoorientado y autocontrolado; es decir, que ya no estará totalmente dirigido por el profesor sino que busca centrarse en el sujeto que aprende. Esto supone subordinar la enseñanza al aprendizaje y reconocer al profesor como mediador del conocimiento y de la cultura social.

- El contexto adquiere una mayor importancia en esta concepción del proceso educativo. Este determina y condiciona en gran medida lo que el estudiante realiza en el aula, la manera en que asume ciertas tareas de aprendizaje, su percepción del estudio y su estilo particular de actuación en la institución educativa.

- Otro factor fundamental es el referido a la interacción entre los sujetos que intervienen en la situación de enseñanza y de aprendizaje en el aula y en nuevos espacios educativos, y el significado de las tareas que realizan de manera conjunta.

- Los dos agentes fundamentales del proceso educativo: el profesor y el estudiante, deben interactuar de manera sistemática en torno a los objetos del conocimiento provenientes de las diferentes disciplinas. Esa interacción está influida por variables de naturaleza cognitiva y afectiva (habilidades, emociones, percepciones, etc.), pertenecientes a ambos sujetos, factores que son movilizados con el propósito fundamental de lograr aprendizajes significativos.

- La necesidad de comprender e instrumentar las operaciones implicadas en la noción de aprender a aprender y acerca de la relación profesor-estudiante-objetos de conocimiento, 
hace necesario recurrir a diversas teorías psicopedagógicas explicativas tanto de los factores que facilitan la interacción entre aquellos, como de otros de tipo individual que, al influir en las modalidades de actuación de los participantes del proceso educativo, determinan las formas en que el estudiante selecciona y utiliza estrategias y procedimientos para lograr aprendizajes significativos.

\section{CONCLUSIONES}

- Los factores económicos y sociales que inciden frecuentemente en los procesos educativos de los estudiantes son la actividad laboral, la dependencia económica, el desempleo, los problemas familiares, el casamiento, los embarazos y la falta de orientación vocacional.

- La influencia de los factores económicos y sociales obliga a los y las estudiantes a no cumplir con sus deberes y tareas en cada una de las materias, priorizando la actividad laboral como alternativa a la solución de los problemas económicos, superando el desempleo y la falta de apoyo familiar.

- La mayor influencia se ve reflejada en que los y las estudiantes no pueden controlar la responsabilidad laboral y el estudio, unido a esto, la situación de los embarazos en las jóvenes, la problemática familiar y por último lo referido a la poca orientación vocacional recibida, afecta el desarrollo del aprendizaje de los estudiantes y dejan sus estudios para otro tiempo.

- La planificación curricular realizada por los docentes considera actividades extracurriculares que los estudiantes incipientemente cumplen por el costo de los trabajos o el tiempo de dedicación a las tareas. A pesar que consideran importante desarrollar planes remediales para los estudiantes, estos no se aplican oportunamente.

\section{REFERENCIAS}

Alegría, Rodrigo; Terán, Rosa; (2005); Metodología de la investigación científica, Instituto de Post Grado y Educación Continua de la Facultad de Filosofía, Letras y Ciencias de la Educación de la Universidad de Guayaquil.

Alomía, Martha; Vásconez, Grecia; (2006); Metodología y monitoreo de evaluación de proyectos, Instituto de Post Grado y Educación Continua de la Facultad de
Filosofía, Letras y Ciencias de la Educación de la Universidad de Guayaquil.

Andino, Patricio; (2006); Metodología de la investigación, Instituto de Post Grado y Educación Continua de la Facultad de Filosofía, Letras y Ciencias de la Educación de la Universidad de Guayaquil.

Arnaz, J.A. (1981). La planeación curricular. Trillas. México.

Bastidas, Alfredo; (2011); El Plan Estratégico Operativo y de Mejoras; Instituto de Post Grado y Educación Continua de la Facultad de Filosofía, Letras y Ciencias de la Educación de la Universidad de Guayaquil.

Cabrera, Guillermo; (2011); Seguimiento y control institucional; Instituto de Post Grado y Educación Continua de la Facultad de Filosofía, Letras y Ciencias de la Educación de la Universidad de Guayaquil.

De los Santos, V. (s. a) Los procesos de permanencia y abandono escolar en Educación superior rieoci.org/ed-Sup-25p.4 200913 de abril.

Estrella, Andrés; Ortiz, Carlos; (2006); Planificación financiera, Instituto de Post Grado y Educación Continua de la Facultad de Filosofía, Letras y Ciencias de la Educación de la Universidad de Guayaquil.

Lozada, Vicente; Terán, Rosa; Yépez, Edison; Alegría, Rodrigo; (2007); Tutoría de la investigación Científica, Instituto de Post Grado y Educación Continua de la Facultad de Filosofía, Letras y Ciencias de la Educación de la Universidad de Guayaquil.

Molina, Fran; (2006); Planificación estratégica, Instituto de Post Grado y Educación Continua de la Facultad de Filosofía, Letras y Ciencias de la Educación de la Universidad de Guayaquil.

Montenegro, Mario; Barros, Teodoro; (2006); Proyectos de desarrollo, Instituto de Post Grado y Educación Continua de la Facultad de Filosofía, Letras y Ciencias de la Educación de la Universidad de Guayaquil.

Otero, L. Deserción: un referente conceptual y metodológico para su estudio. Documento. Páramo, G. y Correa, C. (1999). Deserción estudiantil universitaria.

Palacios, Galo; Isch, Edgar; (2007); Problemas socio económicos contemporáneos, Instituto de Post Grado y Educación Continua de la Facultad de Filosofía, Letras y Ciencias de la Educación de la Universidad de Guayaquil. 
Salinas, Judith; (2011); La investigación evaluativa; Instituto de Post Grado y Educación Continua de la Facultad de Filosofía, Letras y Ciencias de la Educación de la Universidad de Guayaquil.

Valarezo, Reinaldo; (2011); Gestión Administrativa de la Educación Superior; Instituto de Post Grado y Educación Continua de la Facultad de Filosofía, Letras y Ciencias de la Educación de la Universidad de Guayaquil.

\section{Mención}

La presente publicación ha sido posible gracias al aporte de la Universidad Estatal Península de Santa Elena - UPSE, a través de su Observatorio de Políticas Sociales. 